\title{
(6) OPEN ACCESS Alignment of systematic reviews published in the
Cochrane Database of Systematic Reviews and the
Database of Abstracts and Reviews of Effectiveness
with global burden-of-disease data: a bibliographic
analysis
}

Sze Lin Yoong, ${ }^{1,2,3}$ Alix Hall, ${ }^{2,3}$ Christopher M Williams, ${ }^{1,2,3,4}$ Eliza Skelton, ${ }^{2}$ Christopher Oldmeadow, ${ }^{2,3}$ John Wiggers, ${ }^{1,2,3}$ Chante Karimkhani, ${ }^{5}$ Lindsay N Boyers, ${ }^{6}$ Robert P Dellavalle, ${ }^{7,8,9}$ John Hilton, ${ }^{10}$ Luke Wolfenden $^{1,2,3}$

For numbered affiliations see end of article.

\section{Correspondence to} Dr Sze Lin Yoong, Hunter New England Population Health, Locked Bag 10, Wallsend, NSW 2287, Australia; Serene.Yoong@hnehealth.nsw. gov.au

Received 16 December 2014 Revised 27 February 2015 Accepted 27 March 2015 Published Online First 17 April 2015

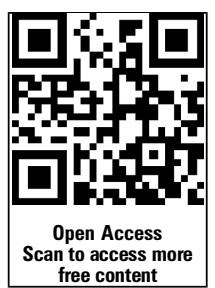

\section{ABSTRACT}

Background Systematic reviews of high-quality evidence are used to inform policy and practice. To improve community health, the production of such reviews should align with burden of disease. This study aims to assess if the volume of research output from systematic reviews proportionally aligns with burden of disease assessed using percentages of mortality and disability-adjusted life years (DALYs).

Methods A cross-sectional audit of reviews published between January 2012 and August 2013 in the Cochrane Database of Systematic Reviews (CDSR) and Database of Abstracts of Reviews of Effects (DARE) was undertaken. Percentages of mortality and DALYs were obtained from the 2010 Global Burden of Disease study. Standardised residual differences (SRD) based on percentages of mortality and DALYs were calculated, where conditions with SRD of more than or less than three were considered overstudied or understudied, respectively.

Results 1029 reviews from CDSR and 1928 reviews from DARE were examined. There was a significant correlation between percentage DALYs and systematic reviews published in CDSR and DARE databases (CDSR: $r=0.68, p=0.001$; DARE: $r=0.60, p<0.001$ ). There was no significant correlation between percentage mortality and number of systematic reviews published in either database (CDSR: $r=0.34, p=0.14$; DARE: $r=0.22$, $\mathrm{p}=0.34$ ). Relative to percentage of mortality, mental and behavioural disorders, musculoskeletal conditions and other non-communicable diseases were overstudied. Maternal disorders were overstudied relative to percentages of mortality and DALYs in CDSR.

Conclusions The focus of systematic reviews is moderately correlated with DALYs. A number of conditions may be overstudied relative to percentage of mortality particularly in the context of health and medical reviews.

\section{INTRODUCTION}

The production of intervention research is key to informing efforts at improving population health. Randomised controlled trials (RCTs) are considered the most internally valid study design and provide the strongest evidence of causal relationships between intervention (exposure) and outcome(s) examined. Observational study designs including cohort and case-control studies also provide useful information particularly in circumstances where it is not feasible to undertake RCTs, such as when the outcome is rare (eg, adverse events), or where longterm follow-up is needed. ${ }^{1}$ Systematic reviews of RCTs represent the highest quality study design in evidence hierarchies. ${ }^{2}$ By integrating and providing global estimates of the available evidence in context of the broader literature, systematic reviews overcome some of the shortcomings of individual studies including systematic bias and statistical imprecision. ${ }^{2}$ While there are some limitations with systematic reviews that can occur as a result of inadequate methodology (eg, poor search strategy) and potential challenges with generalisability and applicability of results, ${ }^{3}$ these reviews are fundamental tools for identifying gaps in the literature, informing evidence-based policy formulation and clinical and public health practice. ${ }^{245}$ Importantly, policymakers increasingly use systematic reviews as the preferred source of evidence to guide decision-making. ${ }^{2}{ }^{6}$

To maximise the benefits of investments in research activity to improve the health and social well-being of the community globally, international organisations including the Institute of Medicine have recommended that government-funded organisations prioritise research activities on the basis of disease burden. ${ }^{7} 8$ Burden of disease is a measure used to assess and compare the relative impact of different diseases and injuries on population health. ${ }^{9}$ Mortality and morbidity (assessed using disability-adjusted life years (DALYs)) are frequently used indices of burden of disease. ${ }^{10}$ Total mortality is defined as the number of deaths due to a specific cause (disease or injury) within the total population. DALYs take into account both years of life lost from premature death and years of life lived with a disability from a condition, providing a measurement of the discrepancy between existing and ideal health status. ${ }^{11}$ Given the role of systematic reviews in facilitating evidence-based health policy and practice, the production of systematic reviews that focus on conditions contributing to greatest health burden is critical for community health improvement. Key producers of systematic 
reviews including Cochrane, ${ }^{12}$ Johanna Briggs Institute $^{13}$ and the Campbell collaboration ${ }^{14}$ have similarly recognised this as an important consideration in generating evidence to best meet global health needs.

Previous studies examining the focus of systematic reviews have found only a modest correlation between the production of research and burden of disease. ${ }^{15}{ }^{16}$ A recent study (2014) examining the representation of specific skin conditions within the Cochrane Database of Systematic reviews (CDSR) found that review output and DALYs were well matched for 5 of the 15 skin conditions examined. ${ }^{17}$ In 2003, an audit of the focus of systematic reviews more broadly found moderate correlation between the number of systematic reviews published in the Cochrane database and the 1990 estimates of DALYs from the Global Burden of Disease (GBD) study $(r=0.54) .{ }^{15}$ This study only described the correlation between the number of reviews and DALYs data and did not attempt to identify specific conditions which have been understudied or overstudied relative to DALYs. Recent estimates from the 2010 GBD study have identified substantial shifts in the leading causes of mortality and DALYs, globally, since 1990. Non-communicable diseases account for $65.5 \%$ of mortality and $54 \%$ of DALYs in 2010 compared with $34 \%$ mortality and 44\% DALYs in $1990 .^{18-20}$

Given such changes, there is a lack of recent evidence assessing the extent to which systematic review output aligns with international estimates of burden of disease. Therefore, this study aimed to (1) examine the correlation between percentage of reviews published in two international databases: CDSR and Database of Abstracts of Review of Effects (DARE) with GBD estimates (mortality and DALYs) from the GBD 2010 study, (2) identify conditions that were overstudied and understudied relative to global mortality and DALYs and (3) assess discrepancies between the databases. CDSR and DARE were chosen because they employ stringent quality measures to ensure that included reviews are of high rigour and are thus recognised as the primary publishers of high-quality systematic reviews in health and medical research.

\section{METHODS}

\section{Data source}

All authors, titles and abstracts of full reviews published in CDSR and DARE between January 2012 and August 2013 were extracted. These dates were chosen to allow for potential diffusion of these new estimates for the GBD 2010 study. Both CDSR and DARE include systematic reviews that evaluate the effects of healthcare interventions and delivery of health services. The production of systematic reviews in CDSR is carried out by 53 Cochrane Review Groups, with over 26000 contributors from over 120 countries. $^{21}$ DARE includes systematic reviews not carried out by Cochrane. Reviews published in the broader literature rated as high quality and of sufficient methodological rigour are selected and assembled by the Centre for Reviews and Dissemination at the University of York, UK.

\section{Data extraction}

Conditions/diseases examined

The types of conditions and diseases examined were coded according to the classifications used in the GBD 2010 study. The study is a systematic and epidemiological dataset produced by the Institute of Health Metrics and Evaluation since 1990, and provides ongoing estimates of mortality and morbidity for 291 diseases from 187 developed and developing countries. ${ }^{22}$ Consistent with the GBD 2010 classifications, ${ }^{23}$ the focus of the reviews was grouped as communicable, non-communicable, injury-related and other (for conditions not included in the taxonomy or not specified). Using a data extraction form, one reviewer (ES) with an honours degree in psychology coded for the broad disease categories and 21 specific conditions (table 1 ). Where the condition was mentioned in the title or abstract as the main focus of the review, this was coded into the corresponding condition. Where the disease category did not directly match a listed category in the GBD 2010, the International Classification of Diseases 10th revision (ICD-10) codes were obtained and matched with the list of ICD-10 codes mapped to the GBD cause list $^{23}$ and coded into the corresponding category. If the study examined more than one specific condition as the main focus of the review, all examined conditions were coded. Where there were uncertainties in coding, it was discussed with a second reviewer (SLY or LW) with a PhD in behavioural sciences and more than 5 years of research and/or health promotion experience. A second reviewer (SLY) checked coding of all diseases, and a consensus process was used to resolve any discrepancies.

Table 1 Number and percentage of reviews in CDSR and DARE examining each condition in the Global Burden of disease 2010 study

\begin{tabular}{|c|c|c|c|c|}
\hline \multirow[b]{2}{*}{ Disease } & \multicolumn{2}{|l|}{ CDSR } & \multicolumn{2}{|l|}{ DARE } \\
\hline & $\begin{array}{l}\text { Number of } \\
\text { publications* }\end{array}$ & $\begin{array}{l}\text { Per } \\
\text { cent }\end{array}$ & $\begin{array}{l}\text { Number of } \\
\text { publications* }\end{array}$ & $\begin{array}{l}\text { Per } \\
\text { cent }\end{array}$ \\
\hline Communicable & 199 & 22 & 216 & 13 \\
\hline HIV, AIDS and tuberculosis & 21 & 2.4 & 44 & 2.6 \\
\hline $\begin{array}{l}\text { Diarrhoea, lower } \\
\text { respiratory infections, } \\
\text { meningitis and other } \\
\text { common infectious } \\
\text { diseases }\end{array}$ & 64 & 7.2 & 80 & 4.6 \\
\hline $\begin{array}{l}\text { Neglected tropical } \\
\text { diseases and malaria }\end{array}$ & 11 & 1.2 & 9 & 0.5 \\
\hline Maternal disorders & 34 & 3.8 & 23 & 1.3 \\
\hline Neonatal disorders & 52 & 5.8 & 25 & 15 \\
\hline Nutritional deficiencies & 8 & 0.9 & 3 & 0.2 \\
\hline Other communicable & 9 & 1.0 & 32 & 1.9 \\
\hline Non-communicable & 638 & 71 & 1406 & 81 \\
\hline Neoplasms & 104 & 12 & 363 & 21 \\
\hline $\begin{array}{l}\text { Cardiovascular and } \\
\text { circulatory diseases }\end{array}$ & 93 & 10 & 209 & 12 \\
\hline $\begin{array}{l}\text { Chronic respiratory } \\
\text { diseases }\end{array}$ & 46 & 5.2 & 34 & 2.0 \\
\hline Cirrhosis of the liver & 9 & 1.0 & 19 & 1.1 \\
\hline Digestive diseases & 32 & 3.6 & 88 & 5.1 \\
\hline Neurological disorders & 52 & 5.8 & 68 & 4.0 \\
\hline $\begin{array}{l}\text { Mental and behavioural } \\
\text { disorders }\end{array}$ & 73 & 8.2 & 129 & 7.5 \\
\hline $\begin{array}{l}\text { Diabetes, urogenital, } \\
\text { blood and endocrine } \\
\text { disorders }\end{array}$ & 86 & 9.6 & 185 & 11 \\
\hline Musculoskeletal disorders & 39 & 4.4 & 190 & 11 \\
\hline Other non-communicable & 147 & 16 & 123 & 7.1 \\
\hline Injury & 57 & 6.4 & 104 & 6.0 \\
\hline Transport injuries & 4 & 0.5 & 2 & 0.1 \\
\hline Unintentional injuries & 51 & 5.7 & 93 & 5.4 \\
\hline $\begin{array}{l}\text { Self-harm and } \\
\text { interpersonal violence }\end{array}$ & 2 & 0.2 & 9 & 0.5 \\
\hline War and disaster & 0 & 0 & 0 & 0 \\
\hline
\end{tabular}

${ }^{*}$ Total number more than sum of reviews due to some reviews examining multiple conditions.

CDSR, Cochrane Database of Systematic Review; DARE, Database of Abstracts and Reviews of Effectiveness. 
Burden of disease assessed using mortality and DALYs

Total mortality rates and DALYs from the GBD 2010 were used as a measure of burden of disease, as recommended by WHO. ${ }^{10}$ Mortality and DALY metrics for each of the 21 conditions examined in the GBD 2010 study were obtained using the GBD interactive time plot tool using the search parameters 'time plot', 'DALYs' metric, 'global' place, 'all ages', 'both' sexes, and '\%' units for each condition. ${ }^{20}$ The methods used to generate mortality and DALY statistics in the GBD 2010 estimates have been previously described. ${ }^{19}$

\section{Statistics}

All data analyses were conducted using SAS (V.9.4, SAS Institute, Cary, North Carolina, USA). ${ }^{24}$ Frequencies and percentages of studies were generated for each condition examined in CDSR and DARE. As the data were not normally distributed, the correlation between the ranking order of the number of reviews focusing on each disease condition published in CDSR and DARE, and percentages of mortality and DALYs, was estimated using Spearman's rank correlation coefficient. To assess if the distribution of publications was consistent with the distribution of number of deaths and DALYs for each condition, the observed $(\mathrm{O})$ number of publications for each condition was compared with the expected (E) number of publications based on the proportion of death and DALYs, and assessed for goodness of fit using the Pearson $\chi^{2}$ test. The E count for each condition was calculated by multiplying the total number of publications by the corresponding proportion of deaths or DALYs for that condition. Standardised residual differences $(\mathrm{O}-\mathrm{E}) / \mathrm{E})$ were displayed using a bar chart, where positive numbers on the bar chart indicate conditions where the number of observed studies was greater than the number of expected studies (overstudied), and negative numbers indicate conditions where the number of expected studies was smaller than the number of observed studies (understudied). To identify conditions that were disproportionally studied, conditions with standardised residual differences (SRD) exceeding 3 (or -3 ) were considered statistically significantly overstudied or understudied. This threshold was chosen as it is accounted for multiple testing using a conservative Bonferroni adjustment. To assess whether the discrepancies in observed and expected number of publications were different between the two databases, SRDs from each condition were compared using the paired $t$ test.

\section{RESULTS}

Overall, 1029 reviews from CDSR and 1928 reviews from DARE were published between January 2012 and August 2013. One hundred and forty-eight reviews (14\%) from CDSR and $284(14 \%)$ reviews from DARE did not examine a condition included in the taxonomy and were excluded from the subsequent analysis. Only 56 (5.4\%) publications in CDSR and 4.5\% of publications in DARE contributed to multiple categories.

\section{Number and percentage of studies assessing each condition in CDSR and DARE}

The majority of reviews published in CDSR (71.4\%) and DARE $(81.5 \%)$ examined non-communicable diseases (table 1). The most frequently examined conditions for both databases were neoplasms (11.6\% CDSR; $21.0 \%$ DARE) and cardiovascular and circulatory diseases (10\% CDSR; 12\% DARE).

There was a significant correlation between the number of publications in CDSR and DALYs $(\mathrm{r}=0.68, \mathrm{p}=0.001)$ and DARE and DALYs $(r=0.60, p<0.001)$. No significant correlation was observed between number of reviews and percentage of mortality for CDSR $(r=0.34, p=0.14)$ or DARE $(r=0.22, p=0.34)$.

Figures 1 and 2 show the SRDs of the reviews published in CDSR and DARE. For reviews published in the CDSR (figure 1 ), overstudied conditions (those with a SRD $>3$ ) relative to percentage of mortality, were mental and behavioural disorders $(\mathrm{SRD}=16.7)$, musculoskeletal conditions $(\mathrm{SRD}=13.4)$, other non-communicable disease $(\mathrm{SRD}=10.5)$ and maternal disorders $(\mathrm{SRD}=6.6)$. Maternal disorders were also overstudied relative to percentage of DALYs $(\mathrm{SRD}=4.58)$.

For DARE (figure 2), the conditions overstudied relative to percentage of mortality were musculoskeletal $(S R D=36.9)$, mental and behavioural diseases $(\mathrm{SRD}=16.0)$ and other noncommunicable disease $(\mathrm{SRD}=4.2)$. None of the conditions were understudied relative to percentage of DALYs.

When comparing SRDs between the number of expected minus observed studies between CDSR and DARE for each condition, no significant differences between the databases for DALYs $(p=0.5777)$ or mortality $(p=0.6592)$ were found.

\section{DISCUSSION}

Using burden-of-disease metrics from the GBD 2010 study, this study found a significant correlation between DALYs and systematic reviews published in CDSR and DARE in 2012-2013. There was no significant correlation between percentage of mortality and the numbers of systematic reviews published in either database. Mental and behavioural disorders, musculoskeletal conditions and other non-communicable diseases were overstudied relative to percentage of global mortality. Collectively, the study suggests that, for most conditions, production of systematic reviews is well aligned with burden-of-disease estimates of DALYs.

A previous study published by Swingler in 2003 found modest correlation $(\mathrm{r}=0.54)$ between the number of reviews published in CDSR and percentage of DALYs, using estimates from the GBD 1990. The primary causes of DALYs have changed considerably since publication of the 1990 estimates. In 2010, the leading causes of DALYs, globally, were ischaemic heart disease (fourth in 1990, an increase of 29\%), followed by lower respiratory infections (first in 1990, a decrease of 44\%), stroke (fifth in 1990; an increase of 19\%), diarrhoeal diseases (second in 1990; a decrease of 51\%), and HIV/AIDS (33rd in 1990; an increase of 351\%). ${ }^{19}$ Despite such changes, a similar moderate association between reviews published in CDSR and percentage of DALYs was identified in the current study $(\mathrm{r}=0.68) .{ }^{15}$

This study is the first to examine the systematic review output and percentage of mortality, and has found that the number of publications in CDSR and DARE did not correlate with the total percentage of mortality. Such findings are consistent with studies examining association between research funding allocation, where positive correlations with percentage of mortality have not been consistently reported. ${ }^{825-27}$

The higher than expected number of published reviews focussing on mental and behavioural disorders, musculoskeletal disorders and other non-communicable disease relative to mortality may reflect the complexity of these conditions and interventions needed for management and prevention. Relative to other conditions which may be more suited to surgical or pharmacological intervention, a broader and more heterogeneous range of strategies may need to be tested to address behavioural and other non-communicable diseases given the complex aetiology of these conditions. Further, as the effectiveness of these 
mental and behavioural disorders musculoskeletal

other non-communicable diseases maternal disorders neurological disorders diabetes/ urogenital/blood and endocrine disorders digestive diseases unintentional injuries neonatal disorders other communicable diseases neoplasms

chronic respiratory diseases diarrhoea, lower respiratory infection nutritional deficiencies
cirrhosis Non-transmittable diseases and malaria HIV/ AIDS and tuberculosis cardiovascular and circulatory diseases transport injuries
intentional injuries

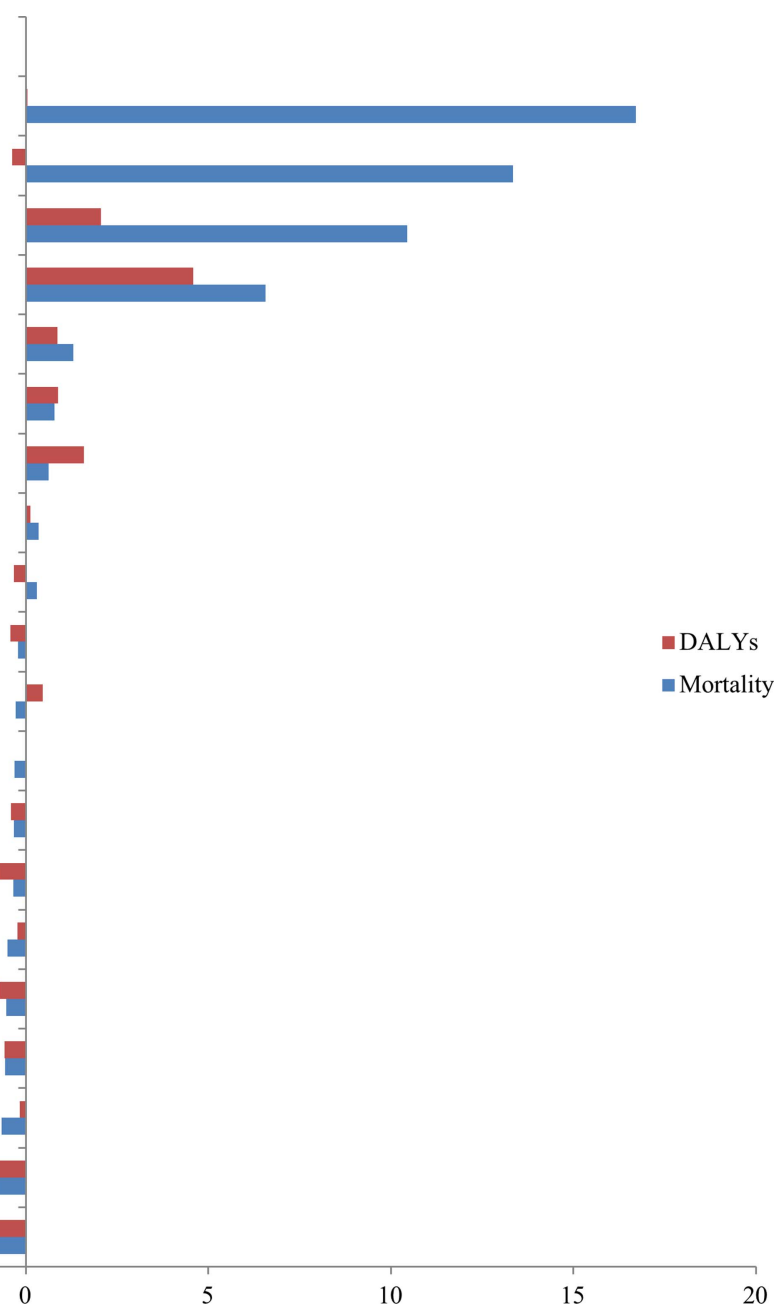

Figure 1 The standardised residual difference ((observed minus expected) /expected) for each cause of mortality and disability published in the Cochrane Database of Systematic Reviews in 2012/2013.

interventions is typically dependent on a range of contextual factors, the need to understand the specific circumstances in which an intervention impacts on health outcomes may have resulted in larger numbers of primary trials and reviews. The specific diseases examined within these conditions are also likely to be the focus of research investment for many higher income countries, and are priority research areas for government and philanthropic sources. ${ }^{28} 29$ As research funding is correlated with publication output, ${ }^{30}$ this is likely to result in an increased number of primary studies and reviews examining these conditions.

For CDSR, specifically, maternal disorders were categorised as overstudied relative to both mortality and DALYs. These findings are consistent with the study by Swingler, which reported that CDSR had a better coverage of maternal issues compared with DARE in $2000 .{ }^{15}$ This could reflect the presence of more established Cochrane Review Groups that support the production of systematic reviews in the area of maternal health. The higher than expected number of reviews in this area could also reflect targeted collaboration with international organisations to achieve specific outcomes as part of the prioritisation process. For example, the Cochrane Pregnancy and Childbirth Review Group worked in collaboration with WHO to update their recommendations for the prevention and treatment of postpartum haemorrhage guidelines in 2012/2013. ${ }^{31}$ Findings from our study suggests that a large number of reviews are produced in this area, and efforts to streamline production of reviews so that they address issues most relevant to areas of need in developed countries may be needed in order to achieve the millennium development goals by $2015 .{ }^{29}$

\section{Strengths and weaknesses of the study}

There are a number of limitations that need to be considered in the interpretation of the study findings. First, although useful, the burden-of-disease estimates including percentage of mortality and DALYs represent just one source of data available to prioritise systematic review activity. Other factors, including the availability of primary studies for review, the number and type of treatment options for a given condition or population, disease trends, emerging evidence that may necessitate production of new reviews, public interest and the presence of disease types which have been established for a longer period of time are among other important considerations that may impact on systematic review output, and are not currently addressed in this manuscript. ${ }^{25} 32$ Second, owing to feasibility constraints, classification was performed using review titles and abstracts only. Examination of full texts may have improved the accuracy of study classification. Further, the inclusion of only reviews 


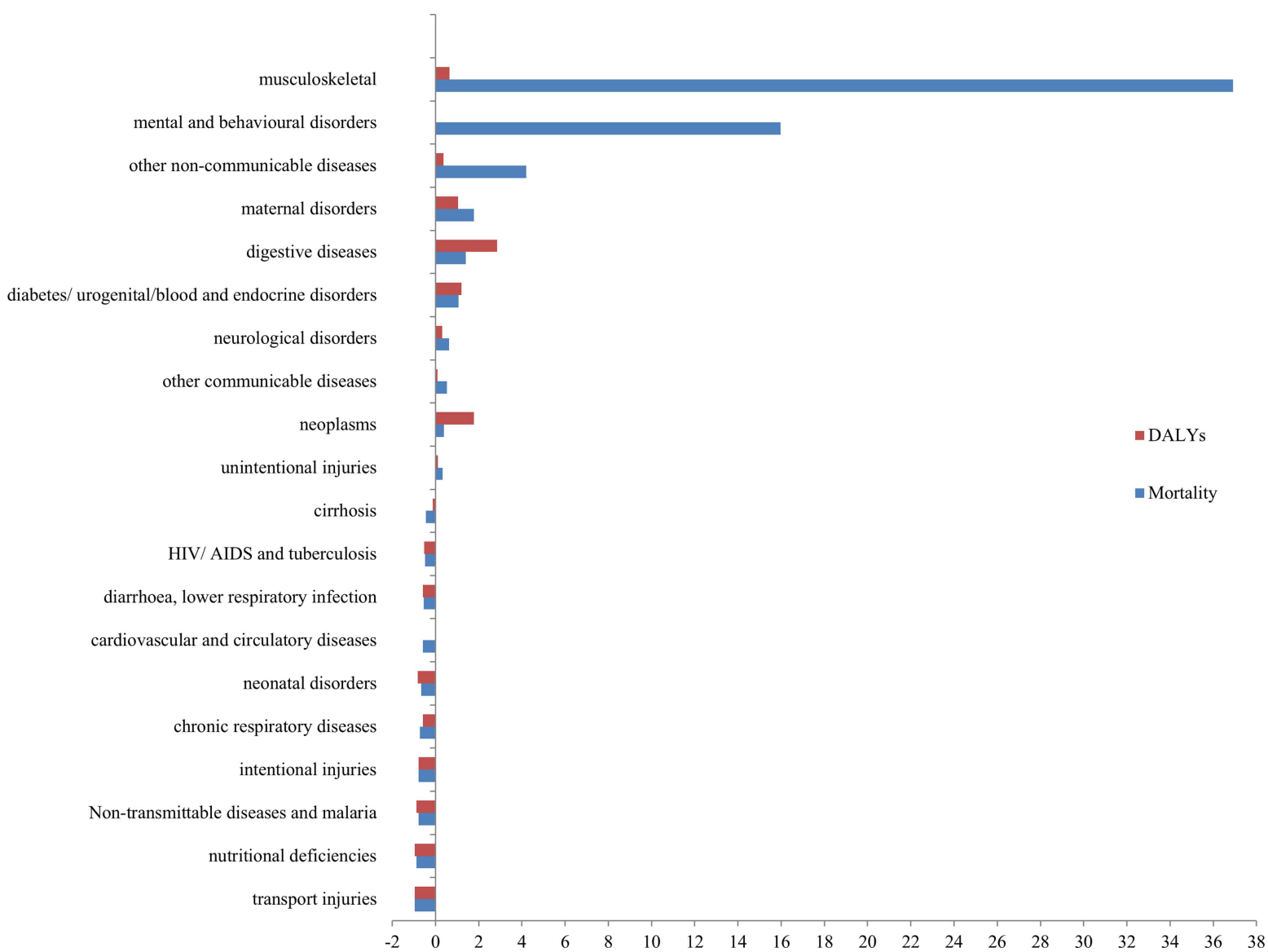

Figure 2 The standardised residual difference ((observed minus expected) /expected) for each cause of mortality and disability published in the Database of Abstracts and Reviews of Effectiveness in 2012/2013.

published between January 2012 and August 2013 may not reflect all reviews available in CDSR and DARE. Third, the data collection method of classifying reviews into a particular condition involved some degree of subjectivity. Fourth, this study was also not powered to specifically identify overstudied or understudied conditions. While we did not identify any understudied conditions, this could have been due to the insufficient number of reviews included in this study. Finally, this study does not take into account the scope of the included review. For example, some authors may include multiple intervention strategies for a condition within one review (lumping), whereas other authors may include only a single intervention in one review, often resulting in multiple reviews for the same condition (splitting).

\section{Unanswered questions and future research}

Our study highlighted that some disease categories may be overstudied in systematic reviews relative to percentages of mortality and DALYs. A closer examination of the specific conditions examined within each broad category of disease is warranted, particularly for conditions identified as overstudied, as this could highlight particular areas for research attention and provide an understanding of potential reasons for the disproportionately larger number of reviews for these conditions. ${ }^{17} 2633$ For example, an examination of Cochrane and non-Cochrane reviews in the maternal health area found that while there were a substantial number of reviews overall, some gaps still existed, particularly for developing countries. ${ }^{34}$ Further, the production of systematic reviews represents only one proxy of research output and usage. The examination of other indicators of output $^{33}$ and research usage (eg, citation rates) with burden-of-disease indicators may provide a more in-depth understanding of how well research production and use aligns more broadly with burden of disease. Despite its importance, little is known regarding the usefulness of strategies to prioritise production of systematic reviews to best inform practice and policy. ${ }^{32}$ Initiatives undertaken by the Cochrane collaboration to improve prioritisation of systematic reviews, including application of an equity lens to inform agenda setting and research decisions, engagement with relevant stakeholders and policymakers, establishing formal prioritisation partnerships with the James Lind Alliance, and development of priority review topics are commendable. ${ }^{12}{ }^{32}$ Such efforts, however, are often varied and largely driven by individual review groups. ${ }^{32}$ While the current study did not identify any difference between CDSR and DARE in terms of number of reviews and alignment with mortality and DALYs, more formal evaluation of the impact of such initiatives is warranted and will inform our understanding on how to best prioritise research topics examined in systematic reviews.

\section{CONCLUSIONS}

The production of systematic reviews in CDSR and DARE moderately aligns with DALYs but not mortality. This study found that mental and behavioural disorders, musculoskeletal conditions and other non-communicable diseases were overstudied 
relative to percentage of mortality. The current study highlights the potential use of burden-of-disease data to inform systematic review prioritisation processes.

\section{What is already known on this subject}

- Burden-of-disease estimates can be used to inform prioritisation of systematic review output.

- A previous study using burden-of-disease estimates from 1990 found moderate correlation between morbidity (assessed using disability-adjusted life years (DALYs)) and publication of systematic reviews.

- Recent estimates of burden of disease indicate that the top causes of mortality and DALYs have changed considerably in the past two decades.

\section{What this study adds}

- This study examines the systematic review of output alignment with most recent mortality and DALYs estimates, and highlights potential areas that may be understudied and overstudied.

- There was a moderate correlation between the production of systematic reviews and DALYs.

- There was no correlation between production of systematic reviews and burden measured in terms of mortality.

- Mental and behavioural disorders, musculoskeletal conditions and other non-communicable diseases were overstudied in the Cochrane Database of Systematic Reviews and the Database of Abstracts of Reviews of Effects, relative to percentage of mortality.

\section{Author affiliations}

${ }^{1}$ Hunter New England Population Health, Wallsend, New South Wales, Australia

${ }^{2}$ The University of Newcastle, School of Medicine and Public Health, Callaghan, New South Wales, Australia

${ }^{3}$ Hunter Medical Research Institute, New Lambton, New South Wales, Australia

${ }^{4}$ The George Institute for Global Health, Sydney, New South Wales, Australia

${ }^{5}$ Columbia University College of Physicians and Surgeons, New York, New York, USA

${ }^{6}$ Georgetown University School of Medicine, Washington DC, USA

${ }^{7}$ Dermatology Service, U.S. Department of Veterans Affairs, Eastern Colorado Health Care System, Denver, Colorado, USA

${ }^{8}$ University of Colorado, School of Medicine, Aurora, Colorado, USA

${ }^{9}$ Department of Epidemiology, Colorado School of Public Health, University of

Colorado, Aurora, Colorado, USA

${ }^{10}$ Cochrane Editorial Unit, The Cochrane Collaboration, London, UK

Twitter Follow John Hilton at @jheditor; Sze Lin Yoong @SereneYoong; Robert Dellavelle @rdellavalle

Contributors SLY and LW contributed to the study design and data collection protocol. SLY, ES and LW collected the data. SLY, LW, CO, CW and JW contributed to the development of statistical plan. $\mathrm{CO}$ analysed the data, and revised the manuscript. AH, CW, JW, CK, LB and JH contributed to revision of the manuscript and interpretation of the results. SLY and LW wrote the manuscript. LW is the guarantor. All the authors had full access to all the data in the study and take responsibility for the integrity of the data and the accuracy of the data analysis.

Funding This work was supported by the Hunter Medical Research Institute and Hunter New England Population Health through providing infrastructure support. LW is supported by an Australian National and Medical Research Council Career Development Fellowship APP1041867. RPD is supported by a salary from the US
Department of Veterans Affairs and grants from the Centers for Disease Control and Prevention (CDC 3U48DP001938-04S1 (PI:Crane), and the National Institutes of Health (NCI R21 CA173654 (PI:Walkocz). JH is an employee of The Cochrane Collaboration which produces the Cochrane Database of Systematic Reviews.

Competing interests None declared.

Provenance and peer review Not commissioned; externally peer reviewed.

Open Access This is an Open Access article distributed in accordance with the Creative Commons Attribution Non Commercial (CC BY-NC 4.0) license, which permits others to distribute, remix, adapt, build upon this work non-commercially, and license their derivative works on different terms, provided the original work is properly cited and the use is non-commercial. See: http://creativecommons.org/ licenses/by-nc/4.0/

\section{REFERENCES}

1 Black N. Why we need observational studies to evaluate the effectiveness of health care. BMJ 1996:312:1215-18.

2 Volmink J, Siegfried N, Robertson K, et al. Research synthesis and dissemination as a bridge to knowledge management: the Cochrane Collaboration. Bull World Health Organ 2004;82:778-83

3 Centre for Reviews and Dissemination. Systematic review: CRD's guidance for undertaking reviews in health care. York: University of York, 2009.

4 Atkins D, Fink K, Slutsky J. Better information for better health care: the Evidence-based Practice Center program and the Agency for Healthcare Research and Quality. Ann Intern Med 2005;142(12 Part 2):1035-41.

5 Lavis JN. How can we support the use of systematic reviews in policymaking? PLoS Med 2009;6:e1000141.

6 Orton L, Lloyd-Williams F, Taylor-Robinson D, et al. The use of research evidence in public health decision making processes: systematic review. PLoS Med 2011;6: e21704.

7 Institute of Medicine Committee on the NIH Research Priority-Setting Process. Scientific opportunities and public needs: improving priority setting and public input at the National Institutes of Health. National Academies Press, 1998.

8 Gross $C P$, Anderson GF, Powe NR. The relation between funding by the National Institutes of Health and the burden of disease. N Engl J Med 1999;340:1881-7.

9 Australian Institute of Health and Welfare. Burden of Disease. Secondary Burden of Disease [Internet]. 2015. http://www.aihw.gov.au/burden-of-disease/

10 World Health Organization. Mortality and global health estimates. http://www.who. int/gho/mortality_burden_disease/en/ (accessed Sep 2014).

11 World Health Organization. Metrics: Disability-Adjusted Life Year (DALY). http:// www.who.int/healthinfo/global_burden_disease/metrics_daly/en/ (accessed Sep 2014).

12 Cochrane Agenda and Priority Setting Methods Group. 2014. https://capsmg. cochrane.org/global-burden-disease-gbd-cochrane-project (accessed Sep 2014).

13 Pearson A, Jordan Z, Munn Z. Translational science and evidence-based healthcare: a clarification and reconceptualization of how knowledge is generated and used in healthcare. Nurs Res Pract 2012;2012:792519.

14 The Campbell Collaboration. About us. http://www.campbellcollaboration.org/ about_us/index.php (accessed Sep 2014).

15 Swingler GH, Volmink J, loannidis J. Number of published systematic reviews and global burden of disease: database analysis. BMJ 2003;327:1083-4.

16 Evans JA, Shim J-M, Ioannidis JPA. Attention to Local Health Burden and the Global Disparity of Health Research. PLOS ONE 2014;9:e90147.

17 Karimkhani C, Boyers LN, Prescott L, et al. Global burden of skin disease as reflected in Cochrane Database of Systematic Reviews. JAMA Dermatol 2014:150:945-51.

18 Lozano R, Naghavi M, Foreman K, et al. Global and regional mortality from 235 causes of death for 20 age groups in 1990 and 2010: a systematic analysis for the Global Burden of Disease Study 2010. Lancet 2013:380:2095-128.

19 Murray CJ, Vos T, Lozano R, et al. Disability-adjusted life years (DALYs) for 291 diseases and injuries in 21 regions, 1990-2010: a systematic analysis for the Global Burden of Disease Study 2010. Lancet 2012:380:2197-223.

20 Institute for Health Metrics and Evaluation (IHME). GBD Cause Patterns. 2014.

21 Allen C, Brurgess M, Clarke M, et al. International activity within Cochrane Review Groups. The Cochrane Collaboration, 2014.

22 Global Burden of Diseases, Injuries, and Risk Factors Study. 2013. http://www. thelancet.com/themed/global-burden-of-disease (accessed Sep 2014).

23 Web table 3- List of International Classification of Diseases codes mapped to the Global Burden of Disease cause list. http://www.healthdata.org/sites/default/files/files/data_ for_download/2012/IHME_GBD2010_CauseListandICD.pdf (accessed Sep 2014).

24 SAS Institute. The SAS system for Windows. Release 9.4. Cary, NC: SAS Institute, 2011. 
25 Gillum LA, Gouveia C, Dorsey ER, et al. NIH disease funding levels and burden of disease. PLOS ONE 2011;6:e16837.

26 Boyers LN, Karimkhani C, Hilton J, et al. Global burden of eye and vision disease as reflected in the Cochrane database of systematic reviews. JAMA Ophthalmol 2015;133:25-31.

27 Lamarre-Cliche M, Castilloux A-M, LeLorier J. Association between the burden of disease and research funding by the Medical Research Council of Canada and the National Institutes of Health. A cross-sectional study. Clin Invest Med 2001;24:83-9.

28 National Health Priority Areas. 2014. https://www.nhmrc.gov.au/grants/researchfunding-statistics-and-data/national-health-priority-areas-nhpas-datasets (accessed Sep 2014).

29 Estimates of Funding for Various Research, Condition, and Disease Categories (RCDC). 2014. http://report.nih.gov/categorical_spending.aspx (accessed Sep 2014).
30 Man J, Weinkauf J, Tsang M, et al. Why do some countries publish more than others? An international comparison of research funding, English proficiency and publication output in highly ranked general medical journals. Eur J Epidemiol 2004;19:811-17.

31 Cochrane Pregnancy and Childbirth Group. WHO announces forthcoming updated guidelines for preventing and treating PPH. 2012. http://pregnancy.cochrane.org/ node/69 (accessed Sep 2014)

32 Nasser $\mathrm{M}$, Welch V, Tugwell $\mathrm{P}$, et al. Ensuring relevance for Cochrane reviews: evaluating processes and methods for prioritizing topics for Cochrane reviews. J Clin Epidemiol 2013;66:474-82.

33 Bourgeois FT, Olson KL, loannidis JP, et al. Association between pediatric clinical trials and global burden of disease. Pediatrics 2014;133:78-87.

34 Chapman E, Reveiz L, Chambliss A, et al. Cochrane systematic reviews are useful to map research gaps for decreasing maternal mortality. I Clin Epidemiol 2013;66:105-12. 